\title{
Mechanistic explanation without the ontic conception
}

\author{
Cory D. Wright
}

Received: 16 April 2011 / Accepted: 24 January 2012 / Published online: 29 March 2012

(C) Springer Science+Business Media B.V. 2012

\begin{abstract}
The ontic conception of scientific explanation has been constructed and motivated on the basis of a putative lexical ambiguity in the term explanation. I raise a puzzle for this ambiguity claim, and then give a deflationary solution under which all ontically-rendered talk of explanation is merely elliptical; what it is elliptical for is a view of scientific explanation that altogether avoids the ontic conception. This result has revisionary consequences for New Mechanists and other philosophers of science, many of whom have assimilated their conception of explanation to the ontic conception.
\end{abstract}

Keywords Ambiguity Craver Exhibition - Mechanism - Mechanistic explanation · Ontic conception $\cdot$ Salmon $\cdot$ Scientific explanation $\cdot$ Syllepsis

The word explanation occurs so continuously, and has so important a place in philosophy, that a little time spent in fixing the meaning of it will be profitably employed.

- John Stuart Mill (1843: 548)

\section{Introduction}

The default sense of the infinitive to explain is a communicative one, pertaining to the transmission of understanding. ${ }^{1}$ Parents explain to their children why their broccoli must be eaten. Students explain that the dog really did eat their homework. Scientists explain how global warming affects arctic ice sheets, or how sensitization of $\mathrm{mGlu}_{2 / 3}$ receptors in the ventral tegmentum 'factors' in nicotine addiction. Like reasons, explanations are given and taken. And like reasoning, explaining some phenomenon $\varphi$ involves operating on internal and/or external representations of $\varphi$ to understand the how or the why of it. Nominalizing explain shifts the focus of attention from

\footnotetext{
${ }^{1}$ Note that the truism that understanding (epistemic) is a norm on good explanation is not impugned by Trout's (2002) lesson that sense of understanding (psychological) is not.

C. D. Wright $(\bowtie)$

Department of Philosophy, California State University, Long Beach, McIntosh Humanities Building 917, 1250 Bellflower Boulevard, Long Beach, CA 90840-2408, USA e-mail: cory.wright@zoho.com
} 
operations to representations; so rather than symbolizing a process, explanation symbolizes the product - a class of complex representations operated upon to generate knowledge and facilitate understanding. It is commonly held that the content of this 'product' can be further separated out from the vehicle. But doing so does not make the semantic structure of explanation any less mind-dependent: explanatory content is always and everywhere the fruit of our cognitive and epistemic labors.

These basic observations are consistent with the mechanistic conception of explanation (MC). According to MC, scientific explanations are explanations in terms of mechanisms. As for mechanisms, the so-called New Mechanists have generally converged on an account, à propos of all sciences, in terms of causal componential systems.

(1) mechanism $={ }_{d f}$ an organized hierarchical system $\mathcal{M}$ of component parts $\left(\pi_{1}, \ldots\right.$, $\left.\pi_{n}\right)$, whose operations $\left(\psi_{1}, \ldots, \psi_{n}\right)$ are coordinated in such a way as to compose a repetitive higher-level activity $\left(\varphi_{i}\right)$ that is ultimately identifiable as a given target phenomenon to be explained.

As for explanation, matters are less straightforward. Perhaps because of their common interests in causality, most New Mechanists have hitched their wagon to Wesley Salmon's ontic conception of scientific explanation (OC). ${ }^{2}$ According to OC, scientific explanations are ontic exhibitions. ${ }^{3}$ The assimilation of these two conceptions results in the thesis that scientific explanations are ontic exhibitions of mechanisms.

(2) Scientific explanations are ontic exhibitions of mechanistic activities fitting into (causal) patterns and regularities.

\footnotetext{
${ }^{2}$ Another reason why is that some New Mechanists have worried that if the default sense of explain is a communicative one, then the only norms governing explanation will be communicative norms (e.g., Craver 2007: ch. 2; see, also Forge 1999: 13). However, this conditional is demonstrably false. Nothing about the meaning of explain itself prevents a conception of explanation from safeguarding the objectivity of explanation, accommodating a role for causal reasoning and its attendant norms, etc. In particular, New Mechanists can accept that causal-mechanical structures in the world provide constraints on explanatory quality without thereby being committed to rendering mechanistic explanations ontically; for example, advocates of a broadly epistemic conception of explanation (EC) can fully and cheerfully adopt less metaphorical versions of Craver's (2007: 26) five norms, and many others besides. So the initial worry is misplaced.

${ }^{3}$ More specifically, the overarching thesis of OC identifies scientific explanations with in re exhibitions of fitness relations between events and (causal) patterns or regularities, such that to explain an event $e$ is to exhibit how $e$ fits into a (causal) pattern or regularity. Craver (personal communication) doubts the fidelity of this formulation. On his reading of Salmon, in re exhibition is irrelevant to OC; instead, what Salmon really meant was that the causal-mechanical patterns and regularities into which events fit are what is explanatory (personal communication). Note, however, that accounts of the causal-mechanical patterns and regularities within which explananda are fit are insufficient to illuminate OC. Such accounts are accounts of the patterns and regularities, and the events or mechanistic activities thereof, that comprise the causal structure of the world. They are not accounts of the fitting of explananda into those patterns, nor accounts of what the in re exhibitions of those fittings are, from which it follows that they are also not yet accounts of scientific explanations according to OC. This point would be question-begging were it not for the fact that the above formulation, which Craver finds dubious, is backed by extensive textual evidence. It recurred, mutatis mutandis, throughout the last 25 years of Salmon's research: e.g., '[OC] sees a scientific explanation as an exhibition of the ways in which what is to be explained fits into natural patterns or regularities in the world' (Salmon 1998: 320; see also, e.g., 1975: 120, 145; 1977: 162; 1978: 700; 1984: 18-19, 121-2, 239, 274, 276; 1989: 86, 93, 121, 134; 1998: 54, 64, 71, 111, 325, 328). Defenders and critics alike have also rehearsed this very formulation of $\mathrm{OC}$, as well as many others that are at most trivially different. Ironically, this includes Craver's own gloss on OC: 'to explain something, one might plausibly argue, just is to show how it fits into the causal structure of the world' (2009: 578). It also includes Glennan, who described OC as the conception that 'explanations exhibit causal mechanisms' (2002: S343 (however, he also misdescribes it - I believe out of charity — as some kind of generic representational realism just a page later)).
} 
Such a thesis reminds me of an old opening salvo of Dennett's (1998: 171): every now and then, good philosophers take up an incurable view and run headlong over the cliff edge. The aim of this paper is to avert further accidents.

Any defense of the thesis that scientific explanations can be reductively identified with ontic exhibitions of mechanisms minimally requires a specification of what it is that explanations are being reductively identified with. Historically, no such specification ever materialized-satisfactory or otherwise. ${ }^{4}$ This would be the end of the story were it not for the fact that (2) has been tidied up by simply excising this strange and feckless construct-EXHIBITION-from Salmon's version of OC. Revisions have led to a more commonly-held assimilation of $\mathrm{OC}$ and $\mathrm{MC}$ according to which an explanans is not so much the ontic exhibition of a mechanism $\mathcal{M}$ - whatever that is but $\mathcal{M}$ itself, while an explanandum just is the (causal) pattern or regularity that its activities are responsible for. Together, $\mathcal{M}$ 's component parts, operations, and organization are themselves what do the explaining, and they do so in virtue of simply producing $\varphi_{i}$.

$\left(2^{\prime}\right)$ Scientific explanations are mechanisms that are responsible for (causal) patterns and regularities.

$\mathrm{OC}$ remains the conceptual mooring for this revised thesis. What $\mathrm{MC}$ contributes to this view is the further identification of the ontic explanation of a given $\varphi$ with the mechanism responsible for it. But it is OC is ultimately in the driver's seat.

This paper uncouples $\mathrm{MC}$ from $\mathrm{OC}$ by undermining a central assumption used to motivate and justify both (2) and ( $\left.2^{\prime}\right)$. My argument is structured as follows. In $\S 2, \mathrm{I}$ observe that many New Mechanists have proclaimed a revised version of OC under which scientific explanations are objective and mind-independent parts of the causal structure of the world. The basis for these proclamations rests upon Salmon's threefold strategy for motivating OC, which I describe in $\S 3$; according to this strategy, OC is motivated by the claim that the term explanation is lexically ambiguous and has a conceptually basic (ontic) sense. After a first pass on the problem with this view in $\S 4$, I raise a puzzle for this ambiguity claim in $\S 5$ : the infinitive to explain and its nominalization seem to pass one important test for lexical ambiguity-namely, syllepsis/conjunction reduction - but, oddly, fails to pass a wide variety of other such tests. In $\S 6$ I argue that the appearance of ambiguity is easily explained away: explanation seems to pass the sylleptic test only because it occurs in constructions involving elliptical techniques and non-literal language. This dissolution to the puzzle suggests that explanation is not ambiguous in the way assumed by, and required for, OC (and, a fortiori, New Mechanists' assimilation of MC to it). Moreover, the dissolution is also consistent with the empirical observation that the default sense of to explain is a communicative one, and yields a view that is less ontologically profligate. I conclude in $\$ 7$ by suggesting that this dissolution allows us to save the mechanistic conception of explanation, which has otherwise become a valuable area of research in philosophy of science, from resting on a mistake.

\footnotetext{
${ }^{4}$ Instead, the term exhibition was merely exchanged with various cognates (e.g., show, reveal, disclose, lay bare, present, demonstrate, indicate, etc.) - cognates which themselves are nothing more than what Craver (2007: 113) aptly called filler terms. This makes it entirely unclear whether anyone-Salmon includedhas ever seriously endorsed $\mathrm{OC}$, or otherwise been rationally committed to it.
} 


\section{The standard view of MC}

It is reasonable to wonder whether this revised thesis in $\left(2^{\prime}\right)$ really has figured centrally in the New Mechanist' Research program. Ultimately, assuaging such skepticism requires settling the prior question of who counts as a New Mechanist. Pending any such head count, there is still a visibly high degree of unity and standardization among discussants of the proper conception of explanation in the literature on $\mathrm{MC}^{5}$ For example, there is Salmon's own claim: '[t]o the extent that causal mechanisms operate, they explain how the world works' (1984: 133), which is repeated almost verbatim by Delehanty: '[... i]t is clear what [mechanisms] are supposed to do for us: they provide causal explanations of the phenomenon of interest' (2005: 721).

Many more examples come from the New Mechanists themselves. 'The behavior of a system at a given level of organization in nature is explained by processes at lower levels and their interactions in the confines of that system', wrote Bechtel (1995: 163). The same point is rehearsed by Craver \& Bechtel, who suggested that 'all higher-level causes are fully explained by constitutive mechanisms' (2007: 548; see also 2006: 473), and then again by Piccinini \& Craver, who 'take it to be uncontroversial' that 'the organized activities of a system's components explain the activities of the whole' (2011: 284 fn. 2). For his part, Craver frequently asserts this standard view in ( $\left.2^{\prime}\right)$. To take just one example, '[...] entities and activities are organized such that they carry out some process, perform some function, or produce some end product. Call this the phenomenon to be explained by the mechanism' (2005: 385, italics subtracted; see also 2006: 356 fn. 1, 359; 2007: 27; 2009: 578). More recently, Darden suggested that such claims are a central plank of the New Mechanist 'team': '[a] mechanism is sought to explain how a phenomenon is produced, how some task is carried out, or how the mechanism as a whole behaves' (2008: 959; see also Machamer 2004: 36). More recently still, Anderson repeats the point, claiming that mechanisms are sought as explanations (forthcoming, fn. 8); her paper is teeming with a variety of expressions of $\left(2^{\prime}\right)$, including suggestions that we 'offer a mechanism as an explanation for why a certain phenomenon occurs $[\ldots]$ ' and proposals to '[...] treat mechanisms as an account of scientific explanation' (forthcoming). Even Glennan has endorsed (2'), his more nuanced formulations of MC notwithstanding: "while it is sometimes the case that a description of the parts of the mechanism will entail a description of the mechanism's outward behavior, the explanation lies not in the logical relationship between these descriptions but in the causal relationships between the parts of the mechanism that produce the behavior described' (2002: S348; see also 2010: 363).

Should we interpret these authors' remarks loosely? Our general preference should be for taking authors at their word, and only engaging in the widespread distribution of attributions of imprecision as necessary. But perhaps the present case is different- do these authors really intend to endorse $\left(2^{\prime}\right)$ ?

\footnotetext{
5 This is not to say that total consensus has been achieved. A few New Mechanists have recently turned to a broadly epistemic conception (see, e.g., Wright 2002; Waskan 2006, 2008; Wright \& Bechtel 2007; Bechtel 2008: 18; Kaplan 2011). But for statements of the view I am describing as standard, see, e.g., Glennan's (1996, 2002, 2005) and Craver's (2007) causal-mechanical accounts of explanation, each of which descends directly and explicitly from OC. See also Forge's $(1986,1998,1999)$ so-called instance conception and Strevens' (2008) so-called kairetic account, both of which are compatible with or sympathetic to the assimilation of MC to OC.
} 
It seems so. Firstly, their remarks are systematic and interlocking, and these two features are grounded in the common commitment to (causal) mechanisms themselves being, eo ipso, scientific explanations. Secondly, New Mechanists often affirm that they really do mean what they say when they say that mechanisms explain. For example - and despite being focused squarely on models and modeling-Glennan wrote: '[t]he mechanism characterized by the mechanical description brings about, and hence explains, the behavior characterized by the behavioral description', adding for good measure that ' $\mathrm{I}$ am adopting an ontic conception of explanation in which the locus of explanatory insight is "in the objects" (2005: $448 \mathrm{fn}$. 5). Similarly,

[w]e will move between claims about mechanistic explanation, and claims about the mechanisms themselves. [...] We agree with Craver that there is at least a sense of explanation that is ontic: that real worldly mechanisms explain their phenomena by producing them. (McKay Illari \& Williamson 2010: 280) ${ }^{6}$

These remarks are not idiosyncratic. As we will continue to see through $\S 4$, they are representative of a genuine trend among New Mechanists and other philosophers of science in which causal-mechanical explanation is standardly conceived as being fundamentally ontic.

Additional exemplifications of New Mechanists' endorsement of $\left(2^{\prime}\right)$ are focused on specific scientific case studies. In discussing the mechanism of neurotransmitter release, Craver asserts that 'the explanandum is the release of one or more quanta of neurotransmitters in the synaptic cleft. The explanans is the mechanism linking the influx of $\mathrm{Ca}^{2+}$ into the axon terminal' (2007: 22). Anderson rehearses a less detailed variant, asking readers to consider the example of 'the mechanism explaining a postsynaptic cell firing', and then writing that 'the firing of a neuron can be explained by providing a mechanism involving a chain of electrochemical events' (2011). For his part, Thagard (2003: $241 \mathrm{ff})$ proposed that biochemical pathways are a kind of mechanism that explain cellular defects leading to disease, while Bechtel \& Abrahamsen wrote that the mechanism of ATP formation, oxidative phosphorylation, explains the 1:3 ratio of oxygen to ATP (2005: 422, cf. $425 \mathrm{fn} .7$ ). In discussing mechanistic accounts of natural selection, Skipper \& Millstein wrote that ' $\mathrm{n}]$ atural selection is a pervasive mechanism that explains adaptations in the living world; it is not merely the explanation of some specific past adaptation event' (2005: 335). The idea has also been invoked in the context of computing mechanisms: '[t]he organized activities of [Turing machines'] components explain the computations they perform' (Piccinini 2007: 507). For his part, Glennan asked, '[i]s there a mechanism that explains the properties of the electromagnetic field?' (1996: 54), while Moss asks 'does the binding of a receptor to a ligand explain or describe?' (2012: 169). Without belaboring the textual evidence further, suffice it to say that these and other such passages seem to express, in one way or another, what has become the standard view.

\footnotetext{
${ }^{6}$ As this passage alludes, many New Mechanists seem torn between stressing the representational role of mechanism sketches, schemas, and models in scientific understanding, on one hand, and steadfastly clutching an ontic rendering of MC on the other. The tension has resulted in a bit of 'code-switching' between ontic and non-ontic construals, as convenient. See, e.g., Glennan (2002), Bechtel \& Abrahamsen (2005), Craver (2005, 2006, 2009), Darden (2008), and Piccinini \& Craver (2011).
} 


\section{Bifurcating explanations: Salmon's threefold strategy}

Given the default sense of explain, the ontic conception may seem counterintuitive. Salmon acknowledged this:

[t]his manner of speaking will sound strange to philosophers who have been strongly influenced by the thesis that explanations are arguments, or by the deeply linguistic approaches that regard explanations as speech acts. In nonphilosophical contexts however, it seems entirely appropriate to say such things as that the gravitational attraction of the moon explains the tides, or the drop in temperature explains the bursting of the pipes. The gravitational attraction and the drop in temperature are out there in the physical world; they are neither linguistic entities (sentences) nor abstract entities (propositions). (1989: 86) ${ }^{7}$

To overcome this counterintuitiveness, and to motivate and justify OC, Salmon pursued the following threefold strategy. ${ }^{8}$ Firstly, show that the term explanation is lexically ambiguous, de facto: i.e., it is just a datum of English that explanation has multiple senses and so can be differentially construed. Secondly, show that disambiguating explanation reveals a multiplicity of senses, at least one of which is the conceptualization of scientific explanations along the lines of OC. Thirdly, show that the ontic sense of explanation is conceptually basic. In virtually all passages where Salmon attempted to motivate and justify OC, the inferential steps of this threefold strategy are more-or-less overt.

With respect to the second step, Salmon took care to articulate the senses of explanation he had in mind. These proved to be just a duality:

[t]he term explanation may be construed in either of two ways. It may be taken as the combination of explanans and explanandum - the explanans having its place in the causal pattern or causal nexus in which it is objectively embedded. This term may also be used to refer to the combination of explanans-statements and explanandum-statement - the linguistic entity that is used to present the objective facts. (1984: 274)

and again,

[p]roponents of [OC] can speak in either of two ways about the relationship between explanations and the world. First, one can say that explanations exist in the world. The explanation of some fact is whatever produced it or brought it about. The explanans consists of certain particular facts and lawful relationships.

\footnotetext{
${ }^{7}$ Salmon was certainly no leading exponent of ordinary language philosophy. So it is rather mystifying why he would seek to motivate OC by appealing to 'non-philosophical contexts'; for it is precisely in such contexts that the commitments of non-literal, unreflective forms of speech are legitimized as 'appropriate'. Why not instead say that the non-philosophical contexts in which misconceived speech seems appropriate are precisely the wrong contexts in which to develop philosophical theories of explanation? Either way, the dispute is not about where to locate gravitational attraction or fluctuations in temperature - so much is obvious; rather, it is about whether explanations are located amongst them.

${ }^{8}$ To his credit, Forge is one of the few advocates of OC to self-critically tackle the issue head on: '[w]hat is it that justifies the claim that an explanation is a state of affairs in the world of something fitting into a pattern?' (1999: 13). Unfortunately, his response was the underwhelming false dichotomy that explanations must be ontic if or because they are not answers.
} 
The explanandum is also some fact. [...] Second, the advocate of [OC] can say that an explanation is something - consisting of sentences or propositions - that reports such facts. (1989: 86)

The general idea is that we can speak of different senses of explanation, which in turn licenses a bifurcation of scientific explanations into two kinds: those that reside in the mind-independent world versus those that reside in the world-dependent mind. Of course, nothing about this general idea settles the question of where scientific explanations ultimately reside; and Salmon was occasionally diffident, suggesting that the choice of one sense over another bottoms out in mere preference or even just linguistic habit (1984: 274), and that, at the very least, both senses are sufficiently viable (1989: 86). However, with respect to the third step, he and other advocates of OC have typically insisted that the ontic sense of explanation is conceptually basic in the theory of explanation, with other senses being derivative. For example, Salmon sympathetically attributed to Coffa the view that 'the linguistic entities that are often called explanations are statements reporting on the actual explanation', since 'what [actually] explains an event is whatever produced it or brought it about' (1989: 133, italics added; see also 1984: 274). In turn, Hooker sympathetically attributed the same view to Salmon, stating that '[s]entential description of the facts follows secondarily [from ontic explanation]', and even added that this third step is 'the right priority for naturalist realism' (1987: 299). For his part, Strevens wrote: '[p]hilosophers sometimes talk as if an explanation were something out in the world, a set of facts to be discovered, and sometimes as if it were a communicative act' (2008: 6); he continued, 'I follow the lead of most philosophers of explanation, and most proponents of the causal approach in particular, in giving the first, ontological sense of explanation precedence. What explains a given phenomenon is a set of causal facts' (ibidem, italics added; see also Glennan 2005: 448). ${ }^{9}$ Together, these three steps prompt the supposition that explanatory 'texts' or models are good to the extent that they adequately represent ontic explanations.

Although many New Mechanists are unified in their endorsement of (2'), few have offered explicit and sustained defenses of Salmon's threefold strategy for establishing OC. One of the richest and most recent, and so which is worth quoting in full, comes from Craver:

[Salmon] defended an ontic view, according to which explanations are objective features of the world. This idea can be brought out by considering an ambiguity in the term explanation. Sometimes explanations are texts-descriptions, models, or representations of any sort that are used to convey information from one person to another. [...] Other times, the term explanation refers to an objective portion of the causal structure of the world, to the set of factors that bring about or sustain a phenomenon (call them objective explanations). What explains the accident? The ice on the road, the whiskey, the argument, the tears, and the severed

\footnotetext{
${ }^{9}$ Strevens's claim about explanantia being sets of causal facts is not what distinguishes his version of OC from the standard view of New Mechanists. For example, although Glennan often speaks of explanantia as being objects, properties, behaviors, and processes, he elsewhere construes explanantia in terms of a factbased ontology: e.g., '[t] he fact that the [generalization about Coke-dispensing machine] is true is explained by facts about how the Coke machine works' (2005: 446). Moreover, this construal harks directly from Salmon himself (1989: 176; see also 1984: 274; Craver 2007: 27; Glennan 2010).
} 
brake cables. There are mechanisms (the objective explanations) and there are their descriptions (explanatory texts). Objective explanations are not texts; they are full-bodied things. They are facts, not representations. They are the kinds of things that are discovered and described. There is no question of objective explanations being 'right' or 'wrong', or 'good' or 'bad'. They just are. Objective explanations, the causes and mechanisms in the world, are the correct starting point in thinking about the criteria for evaluating explanatory texts in neuroscience. [...] Good mechanistic explanatory texts (including prototypes) are good in part because they correctly represent objective explanations. Complete explanatory texts are complete because they represent all and only the relevant portions of the causal structure of the world. Explanatory texts can be accurate enough and complete enough, depending on the pragmatic context in which the explanation is requested and given. Objective explanations are not variable in this way (2007: 27, italics mine)

Craver's invocation of Salmon's threefold strategy is quite plain. Beginning with the unimpeachable distinction between mechanistic representations and represented mechanisms, he then claimed, following Salmon, that the term explanation can and does ambiguously refer to both. From the claim that explanation is ambiguous, he then inferred that explanations are bifurcated into objective explanations and something else, and concluded that mechanistic explanations are best understood as a species of objective explanations. ${ }^{10}$

\section{The problem with the standard view: a first pass}

New Mechanists like Craver often insist that good causal explanations are those that, inter alia, correctly describe the causal structure of the world. I agree. It is surely right to say that mechanistic explanatory texts aim to increase knowledge about mechanisms, and that the successful ones are those whose representations are (cognitively, epistemically) operated on in ways that are truth-conducive-i.e., that 'accurately characterize the causal structure of the world', as he put it (2007: 27 fn. 3, 61). Salmon would have agreed too, having himself supposed that scientific explanation more generally aims at understanding, and that understanding results from knowing how things work (1984: 240; see also Machamer 2004). Obviously, knowledge of how things work is an epistemic matter if anything is, which is just to say that analysis of mechanistic explanatory 'texts' properly requires a broadly epistemic conception of mechanistic explanation.

However, while analyses of the nature of how things work go hand-in-hand with constructing mechanistic explanations, and are crucial for assessing them, surely

\footnotetext{
${ }^{10}$ In addition to distinguishing Craverian objective explanations, which are 'portions of the causal structure of the world', from actual explanations, we should also distinguish them from the objectivity of explanations. Pace Craver, objective explanations are extra-representational; likewise, Salmon's claim that objective relationships hold between genuine explanans and explananda can be interpreted as nothing more than that genuine explanatory relationships are objective. But explanatory objectivity - like truth or maximal accuracy - is primarily a property of representations, and the bifurcation of explanation into objective and something else certainly does not follow from the subjectivity or objectivity of explanations in any epistemic sense.
} 
analyses of what Craver calls 'objective mechanistic explanations' can only be analyses of mechanisms and their constitutive parts, causal processes and interactions, activities and their suboperations, etc. Scientific explanations have properties that no causalmechanical activities have, and vice-versa, which is reflected in the fact that terms like scientific explanation and causal mechanism or causal-mechanical activity are not co-extensional. So prima facie, accounts of scientific explanation that identify them with causal mechanisms or causal-mechanical activities rest on a failure to distinguish the distinct, to properly cleave explanatory structure from causal-mechanical structure. Such identification leads to the mistaken view that scientific explanation designates a class of entities (activities) that enjoy an objective and mind-independent existence, wholly detached from, and metaphysically prior to, the cognitive and epistemic labors of scientists.

So New Mechanists are right to focus on causal structure, but wrong to identify causal structure with norm-governed ratiocinative practices such as explanation (or the products thereof (see Achinstein 1983)). This point has the right consequenceontic explanations cannot be both ontic and explanatory - at the expense of being question-begging. According to (2'), at least some explanations are mechanisms; so it will not do to just assert otherwise, i.e., that the extension of explanation contains no causal-mechanical processes. How then shall we proceed?

The overarching problem with Salmon's threefold strategy is that it proceeds sequentially, but the first step is typically assumed. The assumption is overt in Salmon's own unprompted assertions that 'since explanation is highly ambiguous, it is essential to distinguish a variety of senses', and again, 'the radical ambiguities of explanation create almost endless opportunities for obfuscation and confusion' (1998: 5, 9, italics added). ${ }^{11}$ Many philosophers of science such as Craver, Glennan, Hooker, and Strevens have followed Salmon in supposing that explanations can be bifurcated into objective explanations and something else, and that the bifurcation is justified because the term explanation is lexically ambiguous. ${ }^{12}$ But the bifurcation of scientific explanation, which is necessary to establish OC, is ultimately premised on the ambiguity claim. As this first step is crucial for motivating and justifying theses like (2) or (2'), it cannot just be assumed.

What makes this an interesting issue is not just that the justification of OC rests on linguistic issues, but that it also meets with a curious puzzle (\$5). The rest of the paper offers a dissolution to the puzzle $(\S 6)$, and thus reasons for thinking that New Mechanists would be better off uncoupling their conception of explanation from

\footnotetext{
${ }^{11}$ The assumption might not seem utterly unreasonable; for only if there is more than one sense to spell out in the first place does it make sense to articulate them. But this settles nothing. We want to know what motivates and warrants the assumption that explanation is ambiguous, and this knowledge does not fall out of our agreement that a condition on the possibility of equivocation is that there be multiple senses over which to equivocate in the first place, or that, if by explanation we give voice to the different ways of construing what explanations are, then it appears that explanation is ambiguous, de facto.

${ }^{12}$ For example, Strevens (2008: 6, 319) states that there are two senses or ways of talking about explanation (the ontic way, and some other way (usually epistemic, pragmatic, or cognitive)) and then asserts that most philosophers of explanation give precedence to the former way of speaking. What goes missing are reasons for thinking that these two ways are expressed by the term explanation, de facto, or that the ontic way is conceptually basic and philosophically useful; where there should be an argument, we find merely a preference for a certain style of speech. See also Craver (2007: 27) and McKay Illari \& Williamson (2010: 280).
} 
OC. In particular, I argue that the initial bifurcation can be blocked on the basis of a reminder about the lexical semantics of 'accomplishment' terms like explain and explanation (Achinstein 1983: 15). And if the appearance of ambiguity is only appearance, then explanations are not actually bifurcated - pace Salmon, Forge, Glennan, Craver, Strevens, and others-into objective explanations and something else. In turn, the rationale for construing mechanistic explanations as (ontic exhibitions of) mechanisms is then undercut.

\section{Ambiguity in explanation-ascriptions: a puzzle}

An important battery of tests for lexical ambiguity involves discerning putative cases of zeugma, in which multiple constructions are concurrently governed by a single term (often classed grammatically as noun or verb) that serves as the target of semantic analysis. The most important test for our purposes is syllepsis/conjunction reduction. ${ }^{13}$

Syllepsis/conjunction reduction is a simple function that typically takes as input an ordered pair such as $\{$ the pregnancy was induced, the generalization was induced\}, and returns as output their conjunction and reduction to a single construction (e.g., \{the pregnancy and the generalization were induced $\}$ ). If the output is judged grammatically acceptable, as in (3b),

(3a) Close your \{door/eyes\}, and then go to sleep.

(b) Close your door and your eyes, and then go to sleep.

then the targeted term-here, the directive close - fails the sylleptic test and so is not deemed ambiguous. Like (3b), (4b) seems to fail the test, suggesting that moist is also monosemous.

(4a) Slightly moist \{cake/soil\} is wonderful.

(b) ?Slightly moist cake and soil are wonderful.

Of course, as judgments of grammaticality are scalar, some outputs may only approximate grammatical acceptability (marked by '?'). And so it is certainly possible for the testing to be inconclusive. However, if the output is judged unacceptable (marked by '*'), as in the following examples, then the target term passes the test and the data counts as defeasible evidence for its being ambiguous.

(5a) The \{mistakes/patients\} were committed.

(b) *The mistakes and the patients were committed.

(6a) Firing patterns at the \{neuronal/managerial\} level can be irregular.

(b) *Firing patterns at the neuronal and managerial levels can be irregular.

Given the output of conjoining and reducing the two constructions in (5a), it is immediately clear to competent speakers that were committed is predicated of mistakes and patients in semantically incongruent senses. Likewise, in (6b), the nominal

\footnotetext{
${ }^{13}$ See Zwicky \& Sadock (1975) for a classical paper on tests for ambiguity; see also Sennet (2011) for an overview.
} 
subject firing patterns is ambiguously rendered in terms of electrochemical activation and termination of employment, and the test makes this plain.

Interestingly, the same result appears to obtain for the English infinitive to explain or its nominalization explanation as input. For example,

(7) * The investigator and the oxygen canisters explained why the ValuJet crash in the Florida Everglades occurred.

(8) *The explanation of why the ValuJet crash in the Florida Everglades occurred was given by the investigator and the oxygen canisters.

In (7), the verb explained profiles a particular process type with the same landmark (i.e., the occurrence of the crash) but putatively incongruent trajectors (i.e., the agent described by the investigator and the entities designated by the count noun canisters). ${ }^{14}$ As with (5b), the results seemingly suggest that two different senses of explained are being invoked. The verb explain becomes the nominal subject in (8), and it again appears ambiguous because of the disparate entities posited as explanantia (i.e., investigators and canisters).

That explain and explanation seemingly pass the syllepsis/conjunction reduction test suggests that these terms are lexically ambiguous, just as advocates of OC claim. But this result should strike us as very puzzling, if only because the reasons for attributing lexical ambiguity to the term explanation are otherwise few and weak. For instance, it is not the case that explanation has a single orthographic form but different phonological and semantic poles, as homographs do (e.g., desert/desert, live/live, bass/ bass). Nor does explanation have a univocal phonological structure, but different orthographic and semantic structures, as homophones do (e.g., rap/wrap, break/ brake, aural/oral). Nor is it the case that explanation is homonymous, having the same orthographic and phonetic structure but constituting fully separable and unrelated lexical items (e.g., bark/bark, duck/duck). One such test for homonymy, for instance, checks whether the semantic structure of a target term is modulated by different terms in pairs of syntactically-fixed constructions, as in

(9a) The entire match could not be \{played/lit\} due to the rain.

(b) *The full explanation could not be \{stated/caused $\}$ due to the lack of evidence.

In (9a), both constructions are (nearly) syntactically indistinguishable; match passes the test precisely because of its homonymy, as suggested by the independent sensibility of each construction. But only the first of the two constructions in (9b) is sensible. And the best explanation why explanation fails this test is that it is not homonymous.

The English infinitive and its nominalization explanation are each unambiguous in the sense of having only single lexical entries (corresponding to process and product, respectively). But within those entries, is it not just an empirical fact that explanation is polysemous, having multiple but closely related senses? No. - or, at least, not in any way that would support OC. It is certainly not polysemous in the way that some terms are; e.g., prepositions such as of, around, and over are well-known to have a wide array of senses, even as many as one hundred or more. Nouns (e.g., ring, which includes the

${ }^{14}$ For a preferred overview of linguistic theory and description, see Langacker (2008). 
senses circular mark, circular piece of jewelry, group of people operating together clandestinely, boxing arena, etc.) and verbs (e.g., nurse, which includes senses such as breast-feed, care for the infirm, sip infrequently) often have far fewer senses. Most importantly, even if explain or explanation could be said to be weakly polysemous within their respective lexical entries, (i) none of their senses are ontic, and (ii) what senses they do have are derivative from a basic representational/epistemic one. Confirmation is obtained in virtually any respectable dictionary or historical lexicography. ${ }^{15}$

Additional warrant for the claim that attributions of lexical ambiguity to the term explanation are ill-motivated comes in the form of results from various other tests for ambiguity that contravene those for syllepsis/conjunction reduction. One such test checks for a target term with two or more unrelated negated antonyms (e.g., light in English is ambiguous between not heavy and not dark) or privatives with negated roots (e.g., alethos (remembered, unconcealed) in Greek is ambiguous between apseudos (not false) and atrekês (not disformed), but also between nemertes (faultless), ortos (not duplicitous), etymos (real, actual, authentic)). Another such test is the test of contradiction, which checks for declarative sentences that can be both truly affirmed and truly denied, and licenses an ambiguity claim in cases of truth-value gluts (Zwicky \& Sadock 1975: 7-8). For example, consider

(10a) That bank is not a bank.

(b) Some models are non-models.

(c) *The disease was diagnosed and was not diagnosed.

In $(10 a)$, we can both affirm and deny the construction; we are released from the contradictory result upon disambiguation of the term bank, from which it follows that bank is ambiguous. The same result holds in (10b) for model (particular design, example, fashionista, representation, paragon or ideal specimen, etc.). However, with diagnose, there is no reasonable interpretation by which to affirm $(10 \mathrm{c})$, and hence no truth-value gluts to be cured by disambiguation. Applications of the test of contradiction to explain and explanation are equally revealing.

(10d) *To explain is to not explain.

(e) *No non-explanation is an explanation.

We cannot affirm the sentence in $(10 d)$, and so cannot both affirm it and deny it. The absence of any such truth-value glut indicates that there is no need for disambiguation, which, again, is evidence for the claim that the verb explain is not ambiguous. Nor does explanation appear to admit of multiple senses in (10e).

Lastly, explanation-talk seems to fail yet another kind of test that involves the construction of syllogistic ambiguities. For example,

(11) Her bike wheels are completely true.

(12) What is completely true cannot be false.

$\therefore$ (13) *Her bike wheels cannot be false.

\footnotetext{
${ }^{15}$ Besides the Oxford English Dictionary, see, e.g., the Cambridge International Dictionary of English, or the Random House Unabridged, American Heritage, and Merriam-Webster dictionaries, as well as other lexiographic databases like WordNet or MetaGlossary.
} 
comprise a syllogism involving an equivocation of its major term, wherein the lexical sense of true in (11) has the meaning of being corrected so as to spin along a vertical plane, but has the meaning of being maximally accurate in (12). But again, explanationbeing without homonymous, polysemous, or paranymous senses - is simply an inapposite candidate for passing such a test.

So it seems we have a puzzle. Why does explanation appear to pass a crucial test for lexical ambiguity but then fail to pass so many others? After all, that it passes the test for syllepsis/conjunction reduction would suggest that it would be relatively easy to locate and articulate the different senses that constitute its ambiguous form. But it is not. So what gives?

Advocates of the standard view might respond that we should not think of the data from these linguistic tests as simply fixing all of the facts about lexical sense. Rather, these tests enable understanding and insight about ambiguity, and while some test results may count as (defeasible) evidence in favor of positive or negative claims about ambiguity, they are not necessarily definitive. This is all true. But not only is it ineffectual, the response also cuts both ways. The reliability of a given set of tests is not necessarily lessened just because the evidence that we obtain from it is defeasible; and in any case, our credence in the evidential claims that result from ambiguity tests comes, in part, from statistical demonstrations of their predictive, face, and convergent validity. Moreover, it is precisely because tests for ambiguity do not provide absolute or definitive evidence that we appeal to a large battery of them. Were syllepsis/ conjunction reduction the only test used, we might be subject to false positive results.

In the present case, Salmon's strategy implies that explanation is ambiguously coreferential if explanation can be bifurcated into ontic or objective explanations and something else. If true, we would expect this hypothesis to be overwhelmingly confirmed by an interlocking set of data from a battery of independent and reliable linguistic tests. However, the results are skewed quite far in the opposite direction. Perhaps advocates of the standard view can establish the ambiguity claim in some non-standard way, or can raise doubts about the predictive, face, and convergent validity of linguists' tests (save for syllepsis/conjunction reduction). Until then, it appears that only if explanation passes at least some tests for ambiguity will they have at least some warrant for taking the ambiguity claim to describe a basic datum of English, and thus for initiating Salmon's threefold strategy. And since explanation seems to fail many of them, it very much matters whether or not the result of the one test it does seem to pass is a false positive. In this next section, I argue that the best solution to this puzzle is a dissolution, and that, actually, explanation and its cognates are straightforwardly unambiguous.

\section{Syllepsis via ellipsis: a dissolution}

\subsection{The problem with the standard view: a second pass}

Plainly, mechanisms are integral to mechanistic explanations, and so somehow 'figure' in them. Under one description, this is trivially true. Good mechanistic explanations involve good representations of mechanisms, and would be feckless fictions were there no mechanistic activities to represent. Under another, it rests on a mistake; for the sense in which mechanisms themselves integrally figure in mechanistic explanations is just a 
figurative sense. After all, archetypically and literally, an explainer is a person. And so to say that mechanisms explain is merely to speak using metaphors of personification. It is apiece with claiming:

(14a) My car refuses to start.

(b) Your ulcers are clearly telling you to see a doctor.

(c) Her mesocorticolimbic dopamine system will pay a heavy price as she transitions from drug use to drug abuse.

(d) Some viruses trick the cell membrane into swallowing them by using fake identification.

That is, mechanisms explain in precisely the same sense that cars are moody or have poor etiquette, that $\mathrm{DA}_{1}$ systems beg, borrow, and steal, or that natural selection is a shrewd negotiator. So when New Mechanists propose that a mechanism $\mathcal{M}$ for a phenomenon $\varphi$ explains it, they should not mean that, literally, $\mathcal{M}$ explains $\varphi$. For example, take Thagard's claim that 'biochemical pathways explain by showing how changes within a cell take place as the result of the chemical activities of the molecules that constitute the cell' (2003: 238). No one should interpret Thagard as having literally claimed that biochemical pathways explain or show-much less that those pathways step out for lunch, pass the sodium chloride, or stop to ask for directions. Biochemical pathways, changes in $\mathrm{Ca}^{2+}$ concentrations, phasic $\mathrm{DA}_{1}$ bursting or allostatic mesocorticolimbic dysfunction, oxygen generators, etc. are simply inapposite candidates for doing any explaining. Component parts and component operations constitute mechanisms, not explanations. They do not - cannot - constitute a possible explanans; rather, the relevant parts, operations, and organization of a mechanism $\mathcal{M}$ must always and everywhere, inter alia, be codified into structural or functional representations of some sort.

The dissolution to the puzzle should now be in plain view. If the term explanation passes tests for syllepsis/conjunction reduction, it does so only in virtue of occurring in constructions whose formulation surreptitiously depends on the exploitation of elliptical techniques and the use of non-literal language. This becomes even clearer in examples such as the following:

(15a) Average people think they aren't.

(b) Mechanistic explanations involve mechanisms.

(16a) Average people think they are not average.

(b) Mechanistic explanations involve models and representations of mechanisms.

In (15a), the second instance of average is unarticulated; this is made overt in (16a). The same phenomenon is revealed in comparing (16b) with (15b), wherein representation- and model-talk is shown to have been ellipsed out of the construction. More generally, once we understand the role of ellipsis in formulating these and other such constructions, it becomes evident that the putative 'ambiguity' of explanation is easily explained away. Of course, competent hearers easily navigate the metonymical statements and metaphors of New Mechanists by applying principles of charity and by recognizing that a variety of frequently relied-upon linguistic techniques are being employed, usually for facilitating expository or communicative purposes. This is both normal and unsurprising. What would be utterly surprising is any interpretation of 
advocates of $\mathrm{OC}$ as reflectively committing to a literal or strict rendering of their own view. And this bodes quite poorly for OC. At best, it suggests that OC is not a serious contender; at worst, it suggests that it embodies a rudimentary fallacy - a kind of category mistake, perhaps.

I have been contending that the puzzle dissolves because these terms would not pass the test without certain elliptical techniques and use of non-literal language. In so contending, I have presumed that explain and explanation actually do pass the test for syllepsis/conjunction reduction. But even the presumption may be false; for the test itself has certain intrinsic limitations that cast aspersion on the results that initially seemed favorable to OC. Sennet articulates this nicely:

[g]iven how many parts of speech can be used metaphorically, slavish obedience to the test would postulate massive and unconstrained ambiguity in natural language. The natural answer is to restrict the use of the test to cases in which the words are used literally; but of course the tests are supposed to help us decide when we have literal, semantic difference and when we don't. (2011: §4.8)

So sylleptic tests for lexical ambiguity can be confounded by inputs that are non-literal. In the case of explanation, our dissolution to the puzzle constitutes a good explanation as to why this is. Hence, the problem for which we now have a dissolution may very well have been a pseudo-problem.

\subsection{Further analysis and objections}

In addition to helping us make sense of passages from New Mechanists, this dissolution to the puzzle also makes for good conceptual hygiene. Consider another example. Suppose that $\varphi$ is the occurrence of a runaway high-pitched 'squealing' noise emitted by Joe Strummer's loudspeaker during band practice. What explains $\varphi$ ? Surely the causal story, if anything does; but the causal story and the causes ought not be confused. When Strummer's microphone or guitar pickup begins to couple with the same vibration produced by the loudspeaker, the two components begin to 'ring'. This pure tone resonance causes the loudspeaker to further increase in efficiency, and the microphone or guitar pickup picks it all up again and relays it back to the loudspeaker. The coupling process of sympathetic resonance-a simple causalmechanical activity - is repeated at the speed of sound. But here again, it is quite clear that the causal-mechanical activity is one thing, and the causal-mechanical explanation is something else altogether. The former involves electrical-acoustic properties like vibration and harmonic oscillation, while the latter involves alethic properties like reference and (approximate) truth, epistemic properties like justification, logical properties like conjunction, etc.; the causal-mechanical activity implicates physical wavelengths and positive feedback and guitars and amplifiers, while the causal-mechanical explanation implicates factors like explanatory relevance and abstraction and typological representation. Causal-mechanical explanation has narrative structure, not causal structure.

Suppose the example becomes Strummer's hearing loss, which is caused by the repeated exposure to loud noises. Our dissolution is then open to the following objection. Surely we can also say that Strummer's hearing loss is explained by the 
repeated exposure to loud noises, which just goes to show that the choice of lexeme in constructions such as

(17) The repeated exposure to loud noises \{caused/explains\} Strummer's hearing loss.

makes no semantic difference; rather, the two constructions just say the same thing. And in that case, to say as I have that 'it is quite clear that the causal-mechanical activity is one thing, and the causal-mechanical explanation is another' really does beg the question against OC after all.

In response, observe that the two sentences in (17) do not 'say the same thing'. In the second construction involving explains, the surface grammar hides semantic structure, just as in (15b); and this semantic structure can be made overt, just as in (16b). But even if that were not the case, note that the choice of lexeme also marks a difference in tense. Whereas the first involves the conceptualization of a temporally bounded event prior to the time of utterance, the second involves an atemporal and unbounded event that has held up to the time of utterance and beyond. We could try to eliminate this difference in conceptualization by modifying the constructions to equalize the tense of caused and explains, as in

(18a) Repeated exposure to loud noises \{??causes/explains\} Strummer's hearing loss.

(b) Repeated exposure to loud noises \{caused/?? explained\} Strummer's hearing loss.

However, doing so renders the first sentence of (18a) problematic, since repeated exposure to loud noises is sufficient for Strummer to have hearing loss and additional exposures do not causally overdetermine the same loss. And it also renders the second construction of (18b) less felicitous than (17); for once the repeated exposure were to explain Strummer's hearing loss, it would continue to do so ceteris paribus. Might the same objection hold if we modify aspect instead of tense? Apparently not. A change to the progressive or addition of an auxiliary, as in

(19a) Repeated exposure to loud noises \{is causing/?? is explaining\} Strummer's hearing loss.

(b) Repeated exposure to loud noises \{has caused/??has explained\} Strummer's hearing loss.

again renders the second construction in (19a) and (19b) less felicitous than (17).

So not only can this objection be shaken off, the assimilated view comes in for further drubbing. For example, consider constructions nearly parallel to (7) and (8), such as

*The investigator explained why the ValuJet crash in the Florida Everglades occurred, and so did the oxygen canisters.

(21) *The decreased expression of AMPA/kainate receptors caused the weak EPSPs, and so did the explanation.

and then suppose that explanation in (21) is rendered ontically. A consequence is that the two sets of entities profiled by the two clausal trajectors - i.e., the decrease in 
receptor population density and the explanation, respectively - can be construed as co-extensive participants in the same sort of causal-mechanical relationship. But in that case, they are not obviously semantically incongruent (on an extensionalist semantics); but then, (21) does not obviously pass the test of syllepsis/conjunction reduction. So, the motivation to bifurcate scientific explanations is again undercut.

This inference might be objected to on the grounds that (20) and (21) only sound strange if we decide in advance that explanation is not synonymous with cause or causal mechanism or the like; and yet, this decision is precisely what is up for grabs. In response, this objection is also baseless. Explanation or explain may be closely related in semantic space to a term like cause or causation or mechanism, but they are neither synonymous nor substitutable salva veritate. For example:

(22a) Slipping on a banana peel may \{cause/*explain\} me to fall down the stairs.

(b) The \{cause/??explanation\} of my falling down the stairs could have been prevented.

A better objection is that (21) does sound strange, but that the advocate of OC is not committed to it; instead, he is committed to claims like this one:

* The decreased expression of AMPA/kainate receptors caused the weak EPSPS, and that is the explanation.

And yet, the move to (23) yields no shelter; for the demonstrative phrase that is the explanation anaphorically refers back to the kainate receptors caused the weak EPSPS, which is itself a clause - not an ontic exhibition of a mechanism, or a causal relation, or a repetitive mechanistic activity, or any other such state of affairs.

One final objection is that, in demonstrating that explanation is unambiguous, this paper proves too much; for surely it is possible to stipulatively introduce a new $\tau$-term, explanation, with an overtly ontic sense. If advocates of OC actually do so stipulate, then it follows that the English infinitive and its nominalization really are ambiguous, much like bank, model, or body; and it would then be incorrect to claim, as I have, that mechanistic explanations are not themselves (in re exhibitions of) mechanisms.

This is an interesting issue, which concerns the point that there must be at least one bifurcation of explanations, and so at least two senses of explanation, for any version of $\mathrm{OC}$ to be considered a genuine theoretical rival. ${ }^{16}$ There are several things to mention in response. Firstly, recall that advocates of OC such as Craver and Strevens are not engaged in the project of stipulatively introducing $\tau$-terms (e.g., $\mathcal{M}$-explain); rather, they follow Salmon in taking the ontic sense of explanation to be a genuine and philosophically interesting sense that harks from ordinary street-level or nonphilosophical usage. This paper demonstrates otherwise. In doing so, though, it does not preclude stipulative introduction; for it does not follow from the fact that explanation is unambiguous that it always must be. Secondly, introductions of $\tau$-terms ought to be theoretically useful. But we should take care to distinguish the theoretical pressures of fecundity that motivate such introductions - for example, the reasons why new senses of light or mass or probability were needed - from the speech-act pressures that motivate elliptical or figurative ways of thinking and speaking. In doing so, we stand a better chance of ensuring that a proper metaphysics of explanation does not fall

\footnotetext{
${ }^{16}$ Thanks to an anonymous reviewer for bringing my attention to this point.
} 
victim to reification, hypostasization, and other forms of artifice. In the case at hand, it is not only unnecessary to stipulate that at least one explanation $e$ is the exhibition or activity of $\mathcal{M}$ per (2) or (2'), it is also theoretically unhelpful. We need not superfluously introduce explanation as an additional way to denote hierarchically organized causal systems; we already have perfectly useful terms for so denoting, such as mechanism in (1) or various accounts of causation (and indeed, understanding such systems is where New Mechanists have made their biggest contributions to contemporary philosophy of science). Third and relatedly, we could indeed demonstrate that explanation is ambiguous by stipulatively introducing a $\tau$-term like ontic explanation. But this would amount to nothing more than just stipulating that $\mathrm{OC}$ is true- theft over honest toil.

\section{Auld Lang Syne}

In this paper, I have argued that talk of mechanistic explanations being ontic or in re has been based on an empirically false claim - perpetuated by Salmon and his followersabout the lexical ambiguity of explanation. This is not to say that philosophical contexts should prevent philosophers from uttering sentences like the mechanism of electroacoustical positive feedback is what explains the runaway high-pitched peal emitted by the loudspeaker or repeated exposure to loud noises explains Strummer's hearing loss. More generally, we can even say that effects are explained by their causes, or that causes explain events. But we should also be cognizant that such talk is based on elliptical techniques for ease of exposition. The causally productive in-/ activities of some token $\mathcal{M}$ are just that — extant causally productive in-/activities of $\mathcal{M}$. Explanations are not produced until an explainer contributes her or his epistemic and/or cognitive labor.

Nothing in the very idea of a mechanistic conception of scientific explanation legislates it must be assimilated to OC. And indeed, as I suggested at the outset, what makes (2) incurable is the entailment relation holding between it and $\mathrm{OC}$. Fortunately for MC, it can be safely uncoupled from OC without losing its realist or causal credentials. To see this point better, consider the following three claims.

(24) Our best conceptions of scientific explanation are mechanistic.

(25) Mechanistic conceptions of scientific explanation are ontic.

(26) Ontic conceptions of scientific explanation rest on a mistake.

Claims (24)-(26) do not strictly comprise an inconsistent triad, but they appear to form something near enough. Firstly, we might assert that mechanistic conceptions are our best conceptions of scientific explanation and can be rendered ontically; but it would then be odd to assert that ontic conceptions rest on a mistake. Secondly, we might assert that ontic conceptions rest on a mistake and that mechanistic conceptions are among our very best; but it would then be odd to assert that mechanistic conceptions are to be rendered ontically. Thirdly, we might assert that mechanistic conceptions are to be rendered ontically and that ontic conceptions rest on a mistake; but it would then be odd to assert that mechanistic conceptions are among our very best. 
The appearance of inconsistency dissipates given a doleful willingness to offer up (24)-(26) as the premises of an argument whose conclusion is that all such conceptions are just the best of a bad lot:

(27) Our best conceptions of scientific explanation rest on a mistake.

As the argument for it is unsound, this conclusion is unduly pessimistic. With New Mechanists, I agree that causal-mechanistic conceptions do serve among the best conceptions of scientific explanation currently on offer - a claim I have not argued for here. But contrary to what has become their standard view, the false claim is (25) - not (26). New Mechanists have rightly rallied around Salmon's metaphor that 'the underlying causal mechanisms hold the key to our understanding the world' (1984: 260), but have wrongly taken too seriously its metaphorical entailments, and have mislocated the space of explanations as a result. They have parlayed Salmon's ontic conception, and are worse off because of it.

Acknowledgements Portions of this paper descend from Wright (2002); earlier versions of its arguments were presented at the Central APA in Spring 2004, the Philosophy-Neuroscience-Psychology colloquium at Washington University in St. Louis in Spring 2008 and again at the Center for Logic and Philosophy of Science at Universiteit van Tilburg in Summer 2008. I am grateful to Sorin Bangu, Carl Hoefer, Edouard Machery, Maurice Schouten, and three anonymous reviewers for their constructive criticism.

\section{References}

Achinstein, P. (1983). The nature of explanation. Oxford: Oxford University Press.

Anderson, H. (forthcoming). The case for regularity in mechanistic causal explanation. Synthèse.

Bechtel, W. (1995). Biological and social constraints on cognitive processes: the need for dynamical interactions between levels of organization. Canadian Journal of Philosophy (suppl), 20, 133-164.

Bechtel, W. (2008). Mental mechanisms: Philosophical perspectives on cognitive neuroscience. New York: Routledge.

Bechtel, W. \& Abrahamsen, A. (2005). Explanation: A mechanistic alternative. Studies in History and Philosophy of Biological and Biomedical Sciences, 36, 421-441.

Craver, C. (2005). Beyond reduction: Mechanisms, multifield integration and the unity of neuroscience. Studies in History and Philosophy of Biological and Biomedical Sciences, 36, 373-395.

Craver, C. (2006). When mechanistic models explain. Synthèse, 153, 355-376.

Craver, C. (2007). Explaining the brain: Mechanisms and the mosaic unity of neuroscience. Oxford: Oxford University Press.

Craver, C. (2009). Mechanisms and natural kinds. Philosophical Psychology, 22, 575-594.

Craver, C. \& Bechtel, W. (2006). Mechanism. In S. Sarkar \& J. Pfeifer (Eds.), Philosophy of science: An encyclopedia (pp. 469-478). New York: Routledge.

Craver, C., \& Bechtel, W. (2007). Top-down causation without top-down causes. Biology and Philosophy, $22,547-563$.

Darden, L. (2008). Thinking again about mechanisms. Philosophy of Science, 75, 958-969.

Delehanty, M. (2005). Emergent properties and the context objection to reduction. Biology and Philosophy, 20, 715-734.

Dennett, D. (1998). Brainchildren: Essays on designing minds. Cambridge: MIT Press.

Forge, J. (1986). The instance theory of explanation. Australasian Journal of Philosophy, 64, 127-142.

Forge, J. (1998). Explanation and mechanism: Reflections on the ontic conception of explanation. In D. Anapolitanos, A. Baltas, \& S. Tsinorema (Eds.), Philosophy and the many faces of science (pp. 76-92). Lanham: Rowman \& Littlefield.

Forge, J. (1999). Explanation, quantity and law. Aldershot: Ashgate.

Glennan, S. (1996). Mechanisms and the nature of causation. Erkenntnis, 44, 49-71.

Glennan, S. (2002). Rethinking mechanistic explanation. Philosophy of Science, 69, S342-S353. 
Glennan, S. (2005). Modeling mechanisms. Studies in History and Philosophy of Biological and Biomedical Sciences, 36, 443-464.

Glennan, S. (2010). Mechanisms, causes, and the layered model of the world. Philosophy and Phenomenological Research, 81, 362-381.

Hooker, C. (1987). A Realistic theory of science. Albany: State University of New York Press.

Kaplan, D. (2011). Explanation and description in computational neuroscience. Synthèse, 183, 339-373.

Langacker, R. (2008). Cognitive grammar: A basic introduction. New York: Oxford University Press.

Machamer, P. (2004). Activities and causation: The metaphysics and epistemology of mechanisms. International Studies in the Philosophy of Science, 187, 27-39.

McKay Illari, P. \& Williamson, J. (2010). Function and organization: Comparing the mechanisms of protein synthesis and natural selection. Studies in History and Philosophy of Biological and Biomedical Sciences, 41, 279-291.

Mill, J. S. (1843). A system of logic. London: Harrison \& Co.

Moss, L. (2012). Is the philosophy of mechanism philosophy enough? Studies in History and Philosophy of Biological and Biomedical Sciences, 43, 164-172.

Piccinini, G. (2007). Computing mechanisms. Philosophy of Science, 74, 501-526.

Piccinini, G. \& Craver, C. (2011). Integrating psychology and neuroscience: Functional analyses as mechanism sketches. Synthèse, 183, 283-311.

Salmon, W. (1975). Theoretical explanation and replies to comments. In S. Körner (Ed.), Explanation (pp. 118-145, 160-184). New Haven: Yale University Press.

Salmon, W. (1977). A third dogma of empiricism. In R. Butts \& J. Hintikka (Eds.), Basic problems in methodology and linguistics (pp. 149-166). Dordrecht: Reidel.

Salmon, W. (1978). Why ask, 'Why?' An inquiry concerning scientific explanation. Proceedings and Addresses of the American Philosophical Association, 51, 683-705.

Salmon, W. (1984). Scientific explanation and the causal structure of the world. Princeton: Princeton University Press.

Salmon, W. (1989). Four decades of scientific explanation. Pittsburgh: University of Pittsburgh Press.

Salmon, W. (1998). Causality and explanation. Oxford: Oxford University Press.

Sennet, A. (2011). Ambiguity. In E. Zalta (Ed.), Stanford Encyclopedia of Philosophy. URL $=<\mathrm{http}: / /$ plato.stanford.edu/archives/sum2011/entries/ambiguity/>.

Skipper, R. \& Millstein, R. (2005). Thinking about evolutionary mechanisms: Natural selection. Studies in History and Philosophy of Biological and Biomedical Sciences, 36, 327-347.

Strevens, M. (2008). Depth: An account of scientific explanation. Cambridge, MA: Harvard University Press.

Thagard, P. (2003). Pathways to biomedical discovery. Philosophy of Science, 70, 235-254.

Trout, J. D. (2002). Scientific explanation and sense of understanding. Philosophy of Science, 69, 212-233.

Waskan, J. (2006). Models and cognition: Prediction and explanation in everyday life and in science. Cambridge: MIT Press.

Waskan, J. (2008). Knowledge of counterfactual interventions through cognitive models of mechanisms. International Studies in the Philosophy of Science, 22, 259-275.

Wright, C., \& Bechtel, W. (2007). Mechanisms and psychological explanation. In P. Thagard (Ed.), Handbook of philosophy of psychology and cognitive science (pp. 39-79). New York: Elsevier.

Wright, C. (2002). Reformulating mechanistic explanation. Unpublished manuscript.

Zwicky, A. \& Sadock, J. (1975). Ambiguity tests and how to fail them. Syntax and Semantics, 4, 1-36. 